\title{
Design of a Smart Antenna for Mobile Ad Hoc Network Applications
}

\author{
Marco Di Filippo, Leonardo Lucci, Dania Marabissi, and Stefano Selleri \\ Department of Information Engineering, University of Florence, Via di Santa Marta 3, 50139 Florence, Italy \\ Correspondence should be addressed to Stefano Selleri; stefano.selleri@unifi.it
}

Received 16 June 2014; Accepted 4 July 2014

Academic Editor: Renato Cicchetti

Copyright (C) 2015 Marco Di Filippo et al. This is an open access article distributed under the Creative Commons Attribution License, which permits unrestricted use, distribution, and reproduction in any medium, provided the original work is properly cited.

Among the mobile ad hoc networks appealing characteristics there are network reconfigurability and flexibility. In this context a smart antenna capable of self-configuring multiple high-directivity beams provides a major advantage in terms of power saving, increased range, and spatial reuse of channels. In this paper a smart antenna made of a cylindrical array of patches suitable for MANETs is presented.

\section{Introduction}

Mobile ad hoc networks (MANETs) are wireless networks where nodes can communicate, either directly or indirectly, without any fixed infrastructure (Figure 1) [1,2].

For this reason, MANETs are particularly important in several environments where the fixed infrastructure is not available, not trusted, too expensive, or unreliable. Typical applications are vehicle-to-vehicle communications, emergency services in crisis scenario (earthquake, flood, or fire), tactical communications, and sensor networks.

The lack of a centralized control leads to a self-organizing network working in a distributed manner. Nodes that lie within each other's send range can communicate directly and are responsible for dynamically discovering each other.

In order to enable communication between nodes that are not directly within each other's send range, intermediate nodes act as routers that relay packets generated by other nodes to their destination.

Furthermore, devices are free to join or leave the network and they may move randomly, possibly resulting in rapid and unpredictable topology changes causing link failure and the need of rerouting.

In addition, mobile nodes typically work on batteries, hence have energy limitations, and exhibit great diversity in their range capabilities.

To face these difficulties many research efforts have been done. In particular the use of smart antennas has emerged as an important area of research [3]. Smart antennas can increase the network throughput allowing spatial reuse of the wireless channel and can increase the coverage range and the received signal quality. In addition, they can be used to reduce power emissions thus saving battery energy. Several works in the literature are concerned with how to modify traditional medium access control (MAC) and routing policies to take advantages from smart antennas capabilities.

For example, in [4] a novel MAC protocol based on the use of smart antennas able to separate spatially the users, thus reducing the interference while increasing the throughput, is considered. A unified MAC framework able to operate with different classes of smart antennas is introduced in [5]. While in [6] MAC and routing problems are jointly taken into 


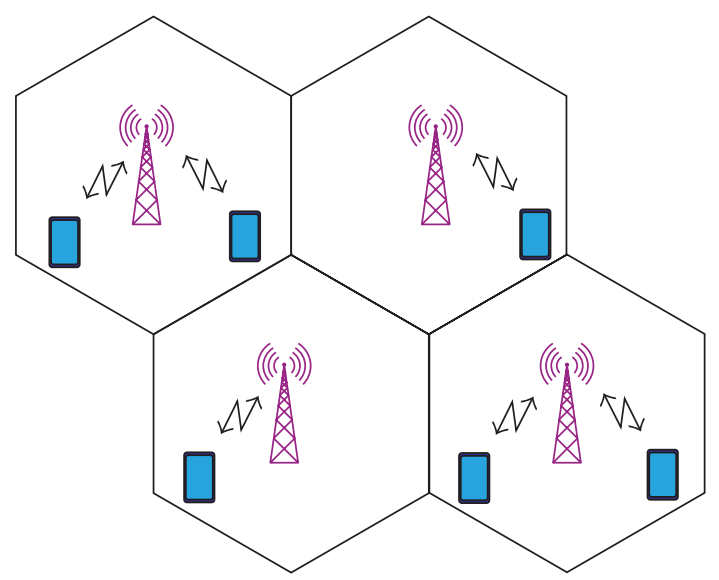

(a)

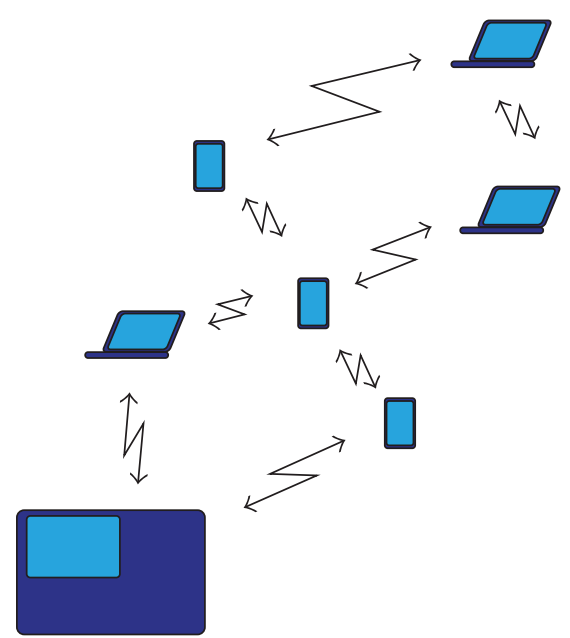

(b)

FIGURE 1: Conventional cellular network with fixed infrastructure (a); and mobile ad hoc network (b).

account using a cross-layer approach that is able to optimally select the communication links performing spatial multiple access, thus enhancing the achievable system throughput.

Particularly interesting is a two-tier MANET architecture with two types of nodes as the one presented in [7]. The higher tier is composed by portable nodes (not mobile or hand-held) with higher computational capabilities that are in key positions to ease the creation and the maintaining of the MANET. These devices are equipped with a smart antenna, with extended range, able to bring up most of the network for its temporary duration, without substantial relocation, hence reducing the issues of changing network topology. The second tier is composed of simpler mobile hand-held devices with single antenna (Figure 2).

Smart antennas are able to project several independent beams, both for interconnection with other base stations and for connection with hand-held devices, thus increasing the spatial reuse.

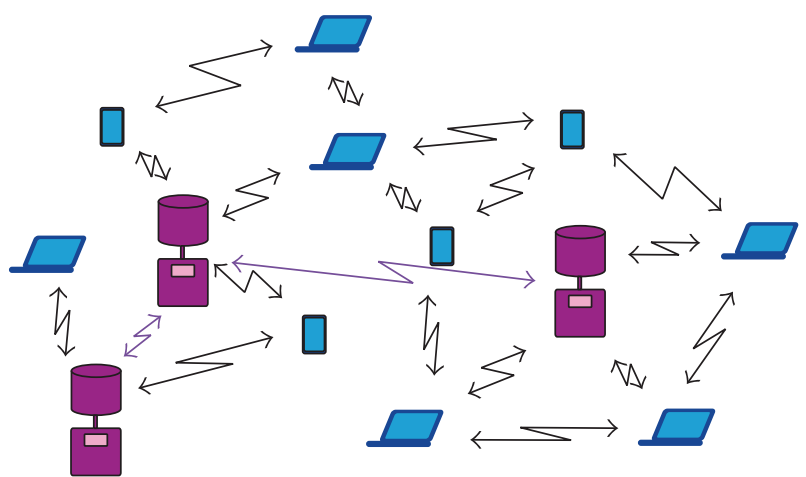

FIgUre 2: Two-tier MANET: purple tier 1 portable nodes and blue tier 2 mobile hand-held devices.

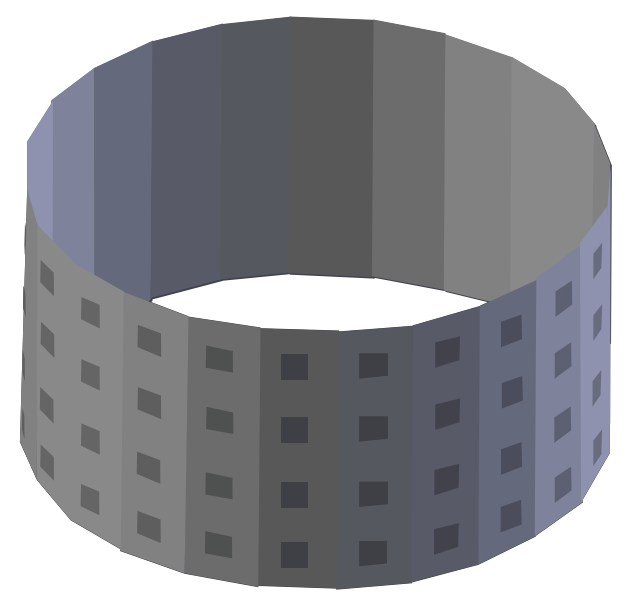

Figure 3: Cylindrical smart array antenna layout.

Taking into account previous considerations, the project of the smart antenna for MANET is of paramount importance, but it has to face several constraints guaranteeing the desired performance.

In this contribution, the design of a cylindrical smart array antenna for a 2-tier MANET working at $5.4 \mathrm{GHz}$ will be presented, focusing on the subarray unit constituting the basic tile of the smart antenna. In particular we focus on tier 1 portable nodes that are responsible for creating the MANET backbone. Section 2 will present the geometry of the antenna and of the tile, while Section 3 will present numerical results. Finally Section 4 will draw some conclusions.

\section{Smart Antenna Configuration}

The basic geometry of the smart array is depicted in Figure 3 . It comprises 24 vertical subarrays, each with four linearly 


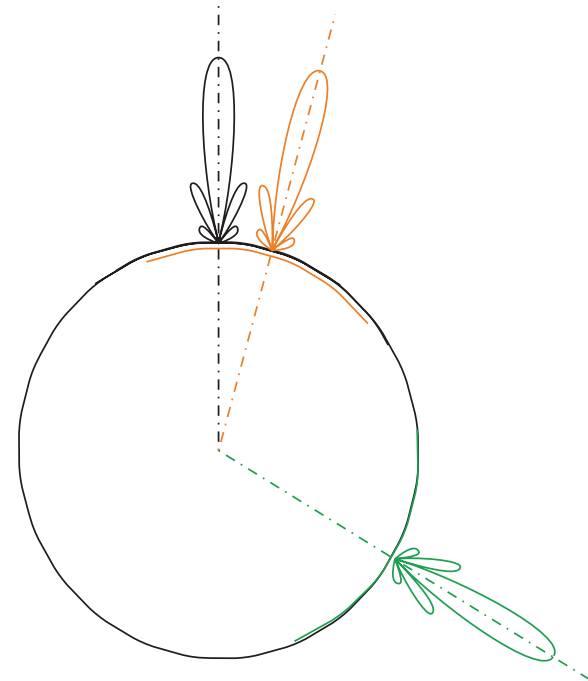

(a)

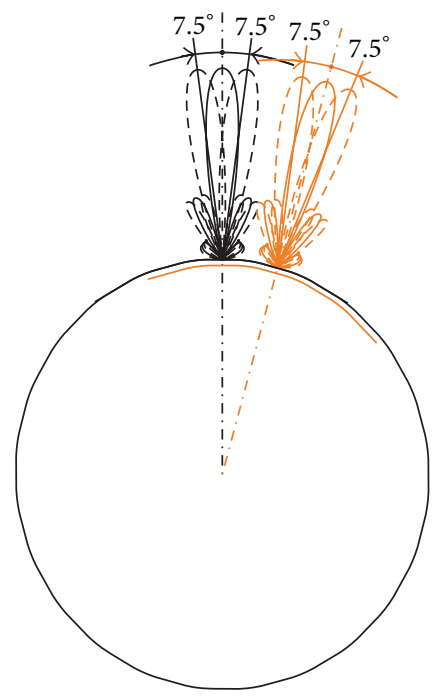

(b)

FIGURE 4: Smart antenna functionality: (a) three possible virtual arrays (black, orange, and green) two of which are largely superimposed; (b) required scan capability from each virtual array to fully scan the horizontal plane.

polarized rectangular patches. At subarray level all patches are fed in phase, leading to a broadside diagram. Phase control of the array is limited to the subarray input line, allowing for beam steering in azimuth.

In principle, only a limited number of subarrays, those facing the desired direction of radiation, can effectively contribute to beam creation. In this project it will be considered that just 5 subarrays will be used at a time for creating each single lobe (Figure 4); these will be called virtual arrays for the given beam. Maximum flexibility would require any group of 5 contiguous subarrays to be selectable at any given time, with close beams being formed by virtual arrays partially superimposing, that is, sharing some physical subarray; hence the smart antenna feeding network needs to provide both the correct phases and the selection of the five subarrays of the virtual array.

Indeed, if 24 subarrays are used, then 24 partially overlapping sets of 5 contiguous subarrays can be defined as the one marked in black and the one marked in orange in Figure 4. Each of these sets needs to scan electronically only $\pm 7.5^{\circ}$. Figure 5 presents a conceptual scheme of the feeding network allowing this maximum flexibility. The $\mathrm{Tx} / \mathrm{Rx}$ modules are $M$ and each one can access independently to any single virtual array. Feeding network complexity is quite high, and simpler layouts can be devised, for example, if no scanning capability of the virtual array is implemented, or if a larger scanning capability is implemented but virtual arrays are nonoverlapping.

To maintain mobility, the antenna is required to be compact; hence a diameter smaller than $40 \mathrm{~cm}$ and a height less than $20 \mathrm{~cm}$ are sought for. By choosing subarrays smaller than $5 \times 20 \mathrm{~cm}$ the requirement is satisfied.

The single subarray is a four-patch printed array with nonuniform elements spacing and nonuniform feeding amplitudes. Relative amplitudes are 1221 , attained via the nonsymmetrical dividers shown in Figure 6, where all the subarray dimensions are given in millimeters. Array is fed via a coaxial cable with a connector mounted beneath the antenna plane in the point shown in Figure 6.

Substrate is Rogers RT/duroid 5880 with relative permittivity $\varepsilon_{r}=2.2$ and $\tan \delta$ in the range [0.0004, 0.0009]. Substrate thickness, chosen among the commercially available, is $t=1,575 \mathrm{~mm}$.

From a technological point of view, possible improvement both in terms of polarization diversity and wide-band operation can be achieved by using radiating patches in planar multilayer technology as presented in [8-10] and/or more complex geometries [11, 12] but for an increased cost and weight.

\section{Numerical Results}

The structure presented was simulated via finite elements (FEM) [13] placing it in a computational box enclosed by perfectly matched layers (PML), so as to correctly take into account the finite ground plane. The obtained $S_{11}$ at the coaxial feed port is reported in Figure 7. Very good isolation between the subarrays has been attained, as Figure 8 shows.

Figure 9 shows the attained patterns for the single subarray, while Figure 10 shows the pattern generated by the virtual array of 5 subarrays, in broadside direction. It is worth noticing that in Figure 10 and subsequent figures two families of graphs are presented. Dashed lines are relative to the case in which the array feed has the exact theoretical value obtained analytically to attain a given direction for the lobe. Solid curves are relative to the realistic case in which phase shifters can synthesize only a discrete set of phases. In this paper phase shifters with a resolution of $45^{\circ}$, hence simple 


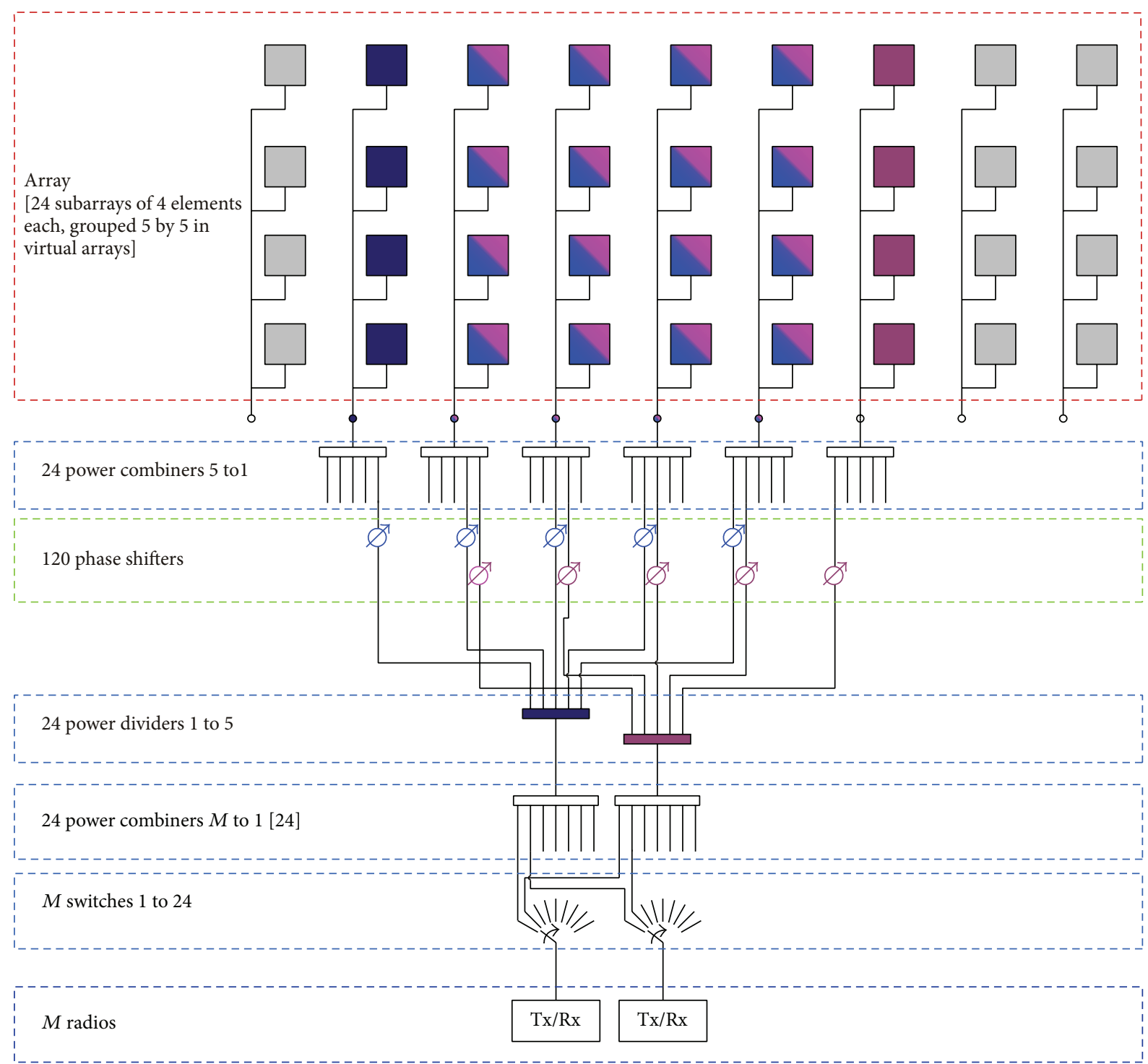

FIGURE 5: Smart antenna feeding network with beam synthesis capabilities.

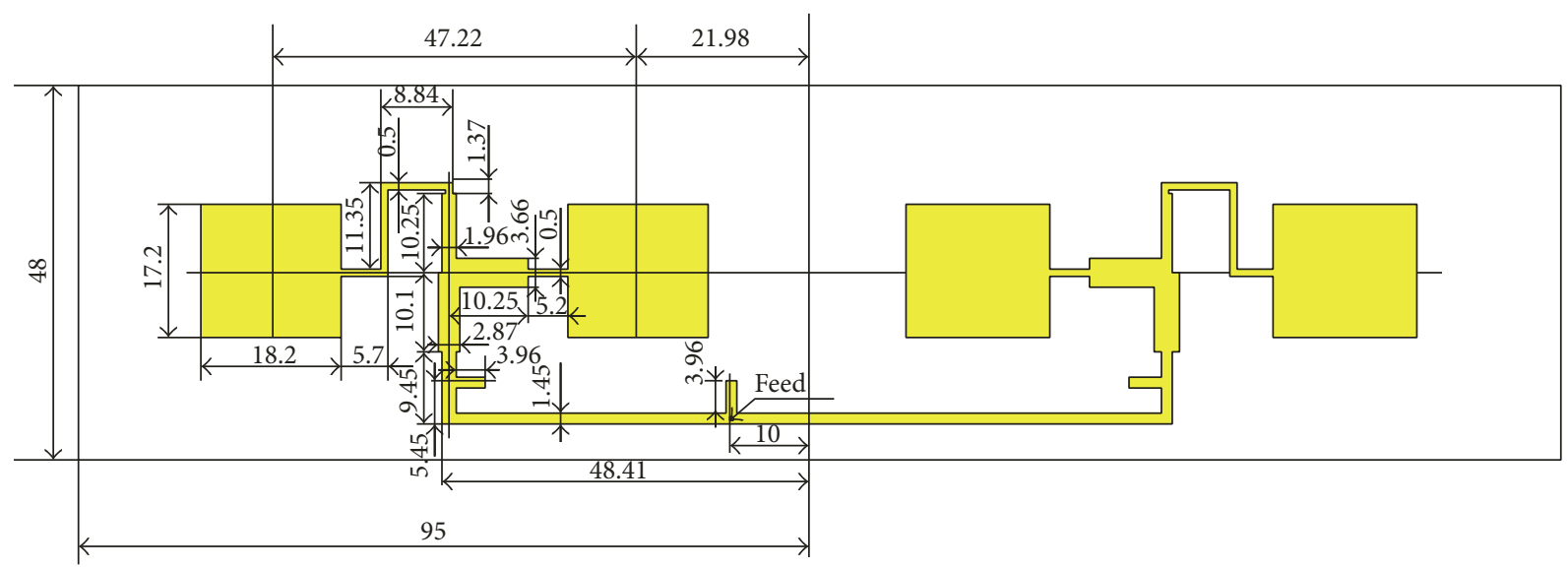

FIgURE 6: Subarray layout and dimensions (mm). 
TABLE 1: Phase shifts for beam steering.

\begin{tabular}{|c|c|c|c|c|c|c|}
\hline & & & Sub -1 & $\begin{array}{l}\text { Central } \\
\text { subarray }\end{array}$ & Sub 1 & \\
\hline \multirow{2}{*}{ Broadside } & Ideal & $152.28^{\circ}$ & $38.62^{\circ}$ & 0 & $38.62^{\circ}$ & $152.28^{\circ}$ \\
\hline & Discrete & $135^{\circ}$ & $45^{\circ}$ & 0 & $45^{\circ}$ & $135^{\circ}$ \\
\hline \multirow{2}{*}{$7.5^{\circ}$} & Ideal & $244.1^{\circ}$ & $89.91^{\circ}$ & $10.1^{\circ}$ & $10.1^{\circ}$ & $89.91^{\circ}$ \\
\hline & Discrete & $225^{\circ}$ & $90^{\circ}$ & 0 & 0 & $90^{\circ}$ \\
\hline \multirow{2}{*}{$36^{\circ}$} & Ideal & $706.9^{\circ}$ & $446.9^{\circ}$ & $235.11^{\circ}$ & $86.45^{\circ}$ & $9.7^{\circ}$ \\
\hline & Discrete & $\begin{array}{c}0^{\circ} \\
\left(720^{\circ}-2 \times 360^{\circ}\right)\end{array}$ & $\begin{array}{c}90^{\circ} \\
\left(460^{\circ}-360^{\circ}\right)\end{array}$ & $225^{\circ}$ & $90^{\circ}$ & 0 \\
\hline
\end{tabular}

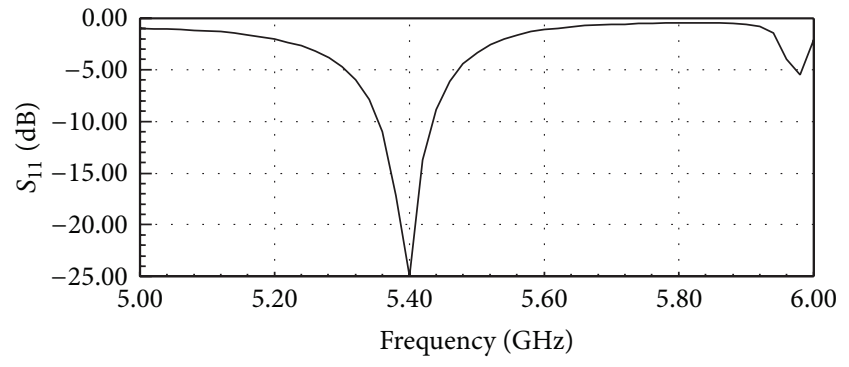

Figure 7: Simulated $S_{11}$ at the subarray coaxial feed port.

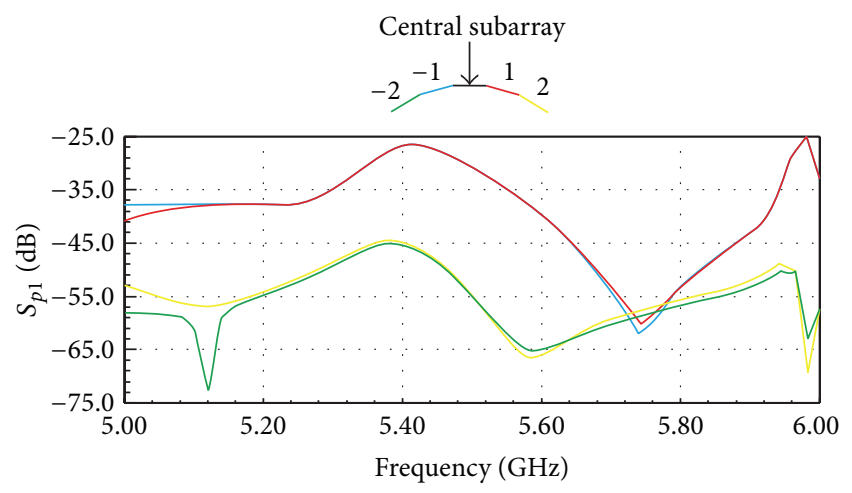

FIGURE 8: Simulated couplings within the virtual arrays, $S_{p 1}$ simulated at the subarray coaxial feed port. Curve colors correspond to relative position on the subarray (above the graph) with respect to the central (black) subarray; red curve is the first subarray to the right, yellow curve the second to the right, and so on.

and economical, are chosen. Table 1 summarizes phase shifts, both analytical and discretized.

Figure 11 shows the pattern for the maximum beam steering required if virtual arrays can overlap, that is, $7.5^{\circ}$. Also in this case two sets of patterns are presented, for ideal and discretized phases, as reported in Table 1 .

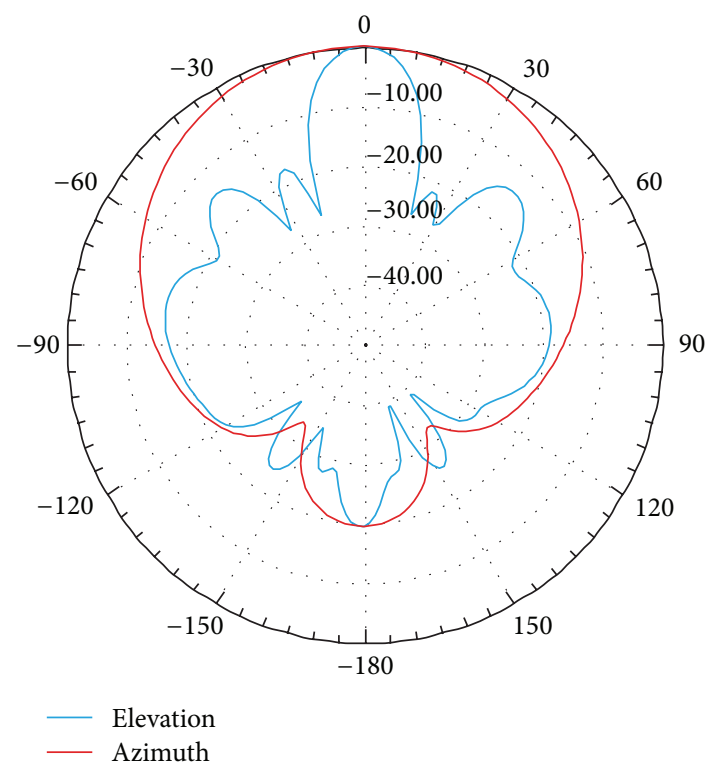

FIGURE 9: Simulated single subarray pattern.

Finally Figure 12 shows the pattern for the maximum beam steering required if virtual arrays cannot overlap, that is, $36^{\circ}$. Also in this case two sets of patterns are presented, for ideal and discretized phases, as reported in Table 1. In this case the grating lobe appearing on the left makes the pattern unusable; hence an overlapping of virtual arrays is mandatory.

\section{Conclusion}

In this contribution a possible layout of smart antenna for MANET applications has been presented. The antenna is capable of producing multiple, high-gain, electronically steered independent beams and is meant to be used in a twotier MANET for the tier 1 nodes responsible for bringing up and maintaining the backbone of the network. 


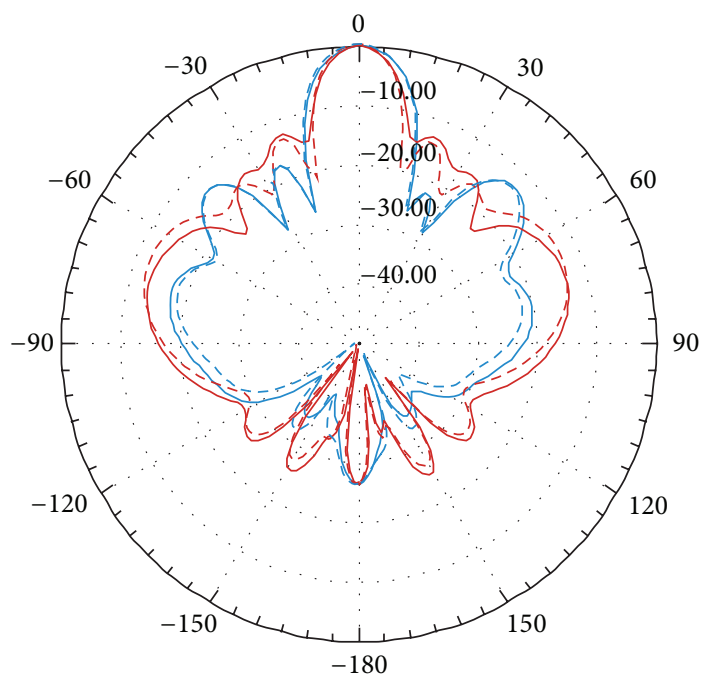

Phases

Ideal Discretized

$--\quad \quad$ Elevation

$--\quad$ Azimuth

Figure 10: Pattern generated by a virtual array of 5 contiguous subarrays on the full cylindrical array (broadside direction). Dashed lines are obtained with ideal phase shifts and solid lines with discretized phase shifts.

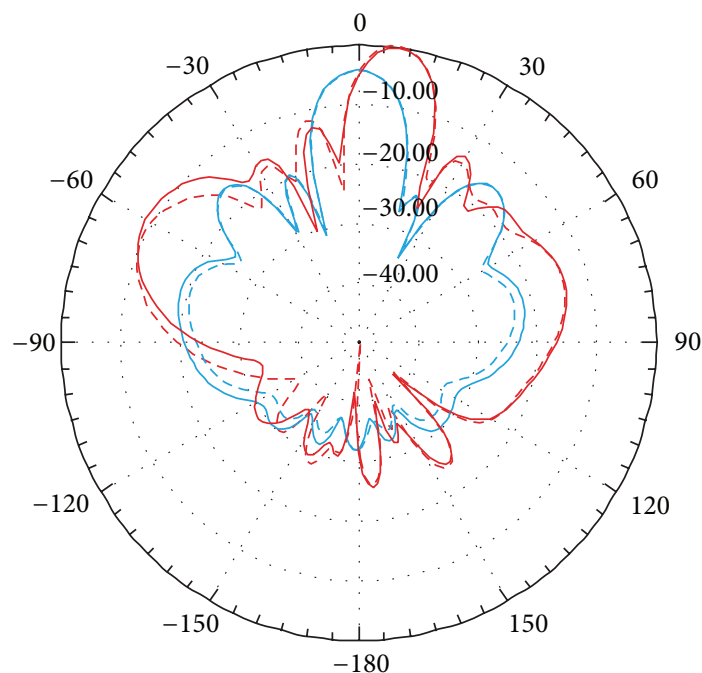

Phases

Ideal Discretized

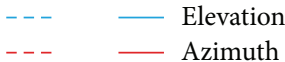

FIGURE 11: Pattern steered by $7.5^{\circ}$ generated by a virtual array of 5 contiguous subarrays on the full cylindrical array. Dashed lines are obtained with ideal phase shifts and solid lines with discretized phase shifts.

Antenna layout has been kept simple to allow for a cheap and lightweight realization. Also to this aim, the usage of cheap and simple phase shifter with a fairly coarse phase discretization did not lead to sensible performance degradation.

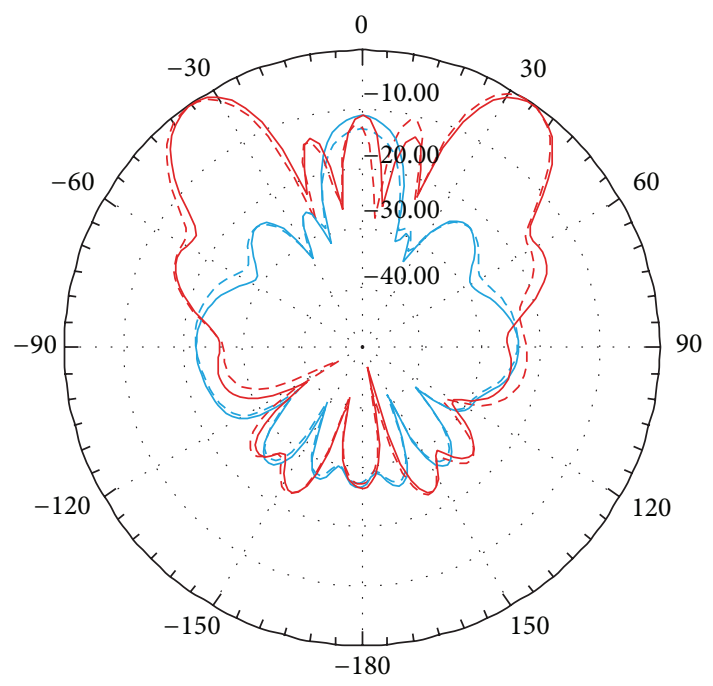

Phases

Ideal Discretized

$--\quad \_$Elevation

$--\quad$ Azimuth

FIgURE 12: Pattern steered by $36^{\circ}$ generated by a virtual array of 5 contiguous subarrays on the full cylindrical array. Dashed lines are obtained with ideal phase shifts, solid lines with discretized phase shifts.

\section{Conflict of Interests}

The authors declare that there is no conflict of interests regarding the publication of this paper. 


\section{References}

[1] M. Conti and S. Giordano, "Mobile ad hoc networking: milestones, challenges, and new research directions," IEEE Communications Magazine, vol. 52, no. 1, pp. 85-96, 2014.

[2] M. Frodigh, P. Johansson, and P. Larsson, "Wireless ad hoc networking: the art of networking without a network," Ericsson Review, no. 4, pp. 248-263, 2000.

[3] J. H. Winters, "Smart antenna techniques and their application to wireless ad hoc networks," IEEE Wireless Communications, vol. 13, no. 4, pp. 77-83, 2006.

[4] M. I. Rafique, "Exploiting smart antennas for spatial reuse and multiplexing in wireless mesh networks," in Proceedings of the 14th IEEE International Symposium on a World of Wireless, Mobile and Multimedia Networks (WoWMoM '13), pp. 1-7, June 2013.

[5] K. Sundaresan and R. Sivakumar, "A unified MAC layer framework for Ad-Hoc networks with smart antennas," IEEE/ACM Transactions on Networking, vol. 15, no. 3, pp. 546-559, 2007.

[6] M. Yazdanpanah, C. Assi, and Y. Shayan, "Optimal joint routing and scheduling in wireless mesh networks with smart antennas," in Proceedings of the IEEE International Symposium on "A World of Wireless, Mobile and Multimedia Networks" (WoWMoM '10), pp. 1-7, June 2010.

[7] N. Funabiki, T. Nakanishi, and K. Watanabe, "An extension of routing tree algorithm for smart antenna use in wireless internet-access mesh network," in Proceedings of the 1st IEEE Global Conference on Consumer Electronics (GCCE '12), pp. 633637, October 2012.

[8] P. Capece, L. Lucci, G. Pelosi, M. Porfilio, M. Righini, and W. Steffè, "A multi-layer PCB dual-polarized antenna demonstrator for future SAR applications," IEEE Antennas and Wireless Propagation Letters, vol. 13, pp. 297-300, 2014.

[9] P. Capece, L. Lucci, G. Pelosi, M. Porfilio, M. Righini, and W. Steffè, "Fully integrated multi-layer power distribution network for SAR dual-polarized linear arrays," IEEE Microwave and Wireless Components Letters. In press.

[10] D. Caratelli, R. Cicchetti, G. Bit-Babik, and A. Faraone, "A perturbed E-shaped patch antenna for wideband WLAN applications," IEEE Transactions on Antennas and Propagation, vol. 54, no. 6, pp. 1871-1874, 2006.

[11] P. Moeikham, C. Mahatthanajatuphat, and P. Akkaraekthalin, "A compact UWB antenna with a quarter-wavelength strip in a rectangular slot for $5.5 \mathrm{GHz}$ band notch," International Journal of Antennas and Propagation, vol. 2013, Article ID 574128, 9 pages, 2013.

[12] R. Gonçalves, P. Pinho, and N. B. Carvalho, "Compact, frequency reconfigurable, printed monopole antenna," International Journal of Antennas and Propagation, vol. 2012, Article ID 602780, 6 pages, 2012.

[13] G. Pelosi, R. Coccioli, and S. Selleri, Quick Finite Elements for Electromagnetic Waves, Artech House, Boston, Mass, USA, 2nd edition, 2009. 

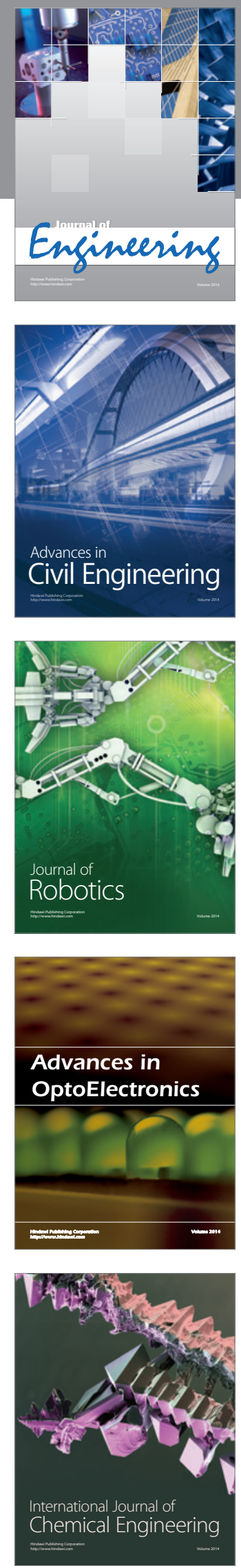

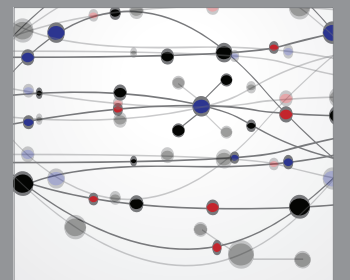

The Scientific World Journal
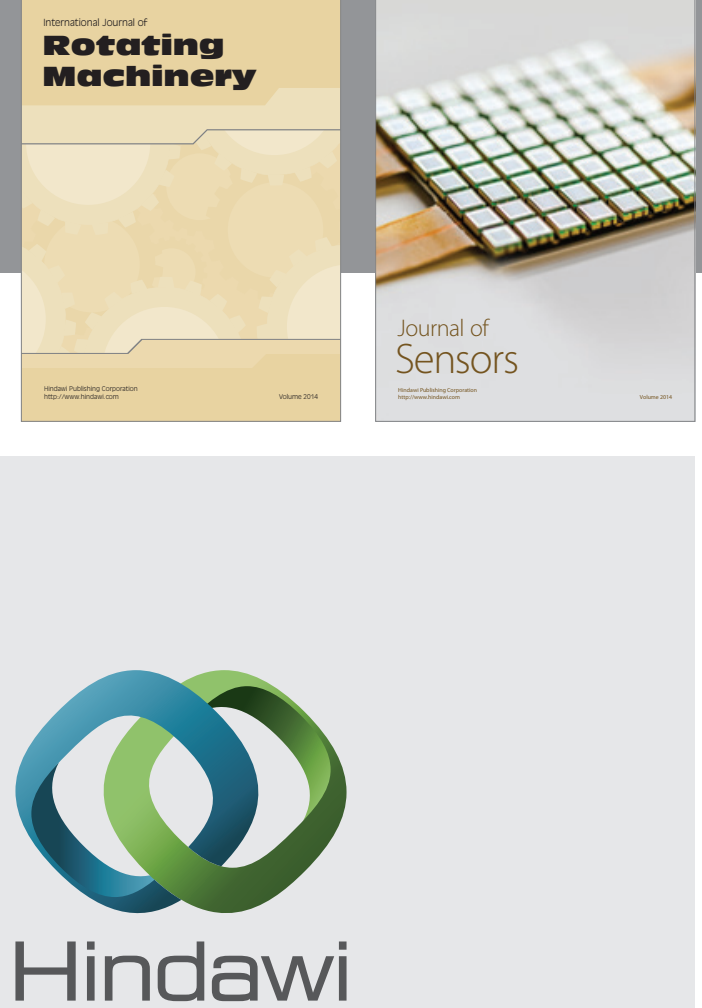

Submit your manuscripts at http://www.hindawi.com
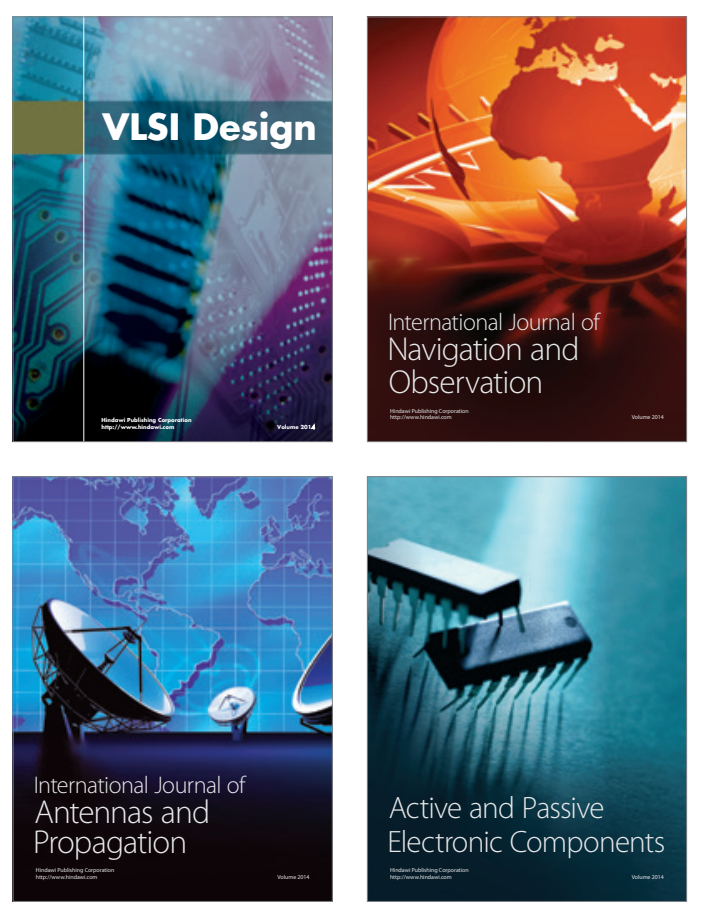
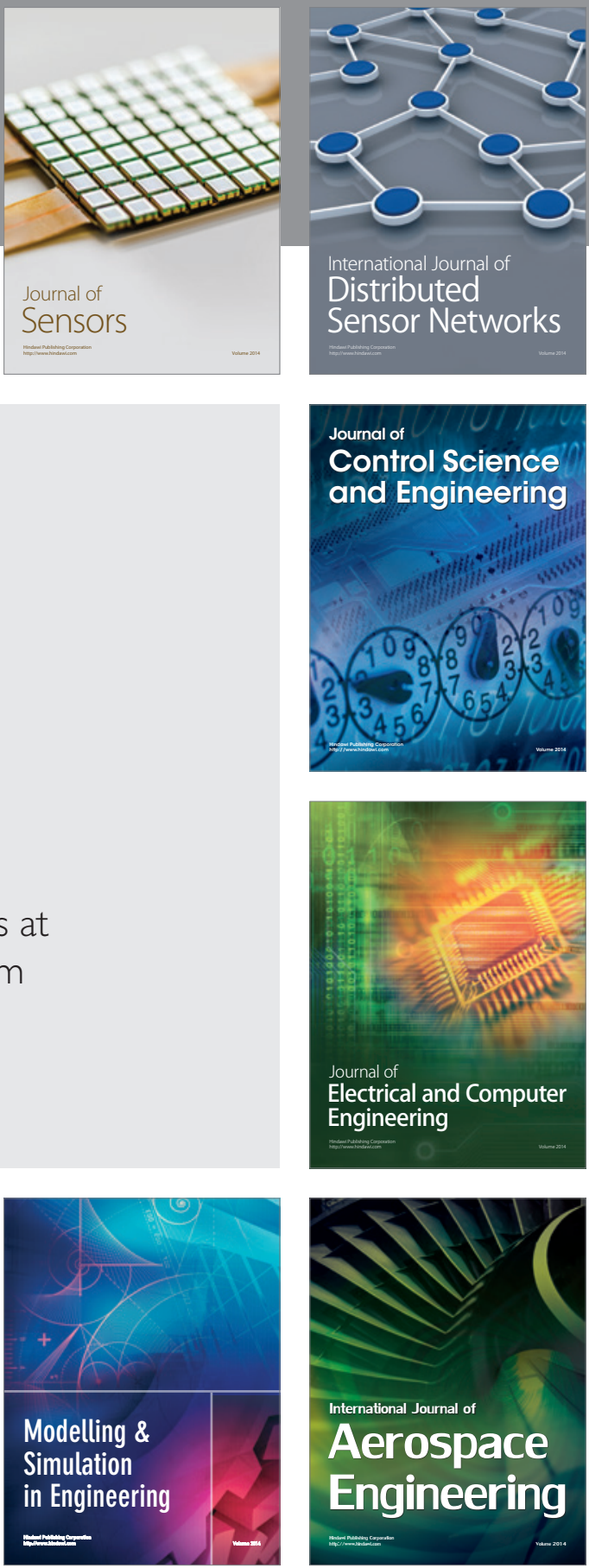

Journal of

Control Science

and Engineering
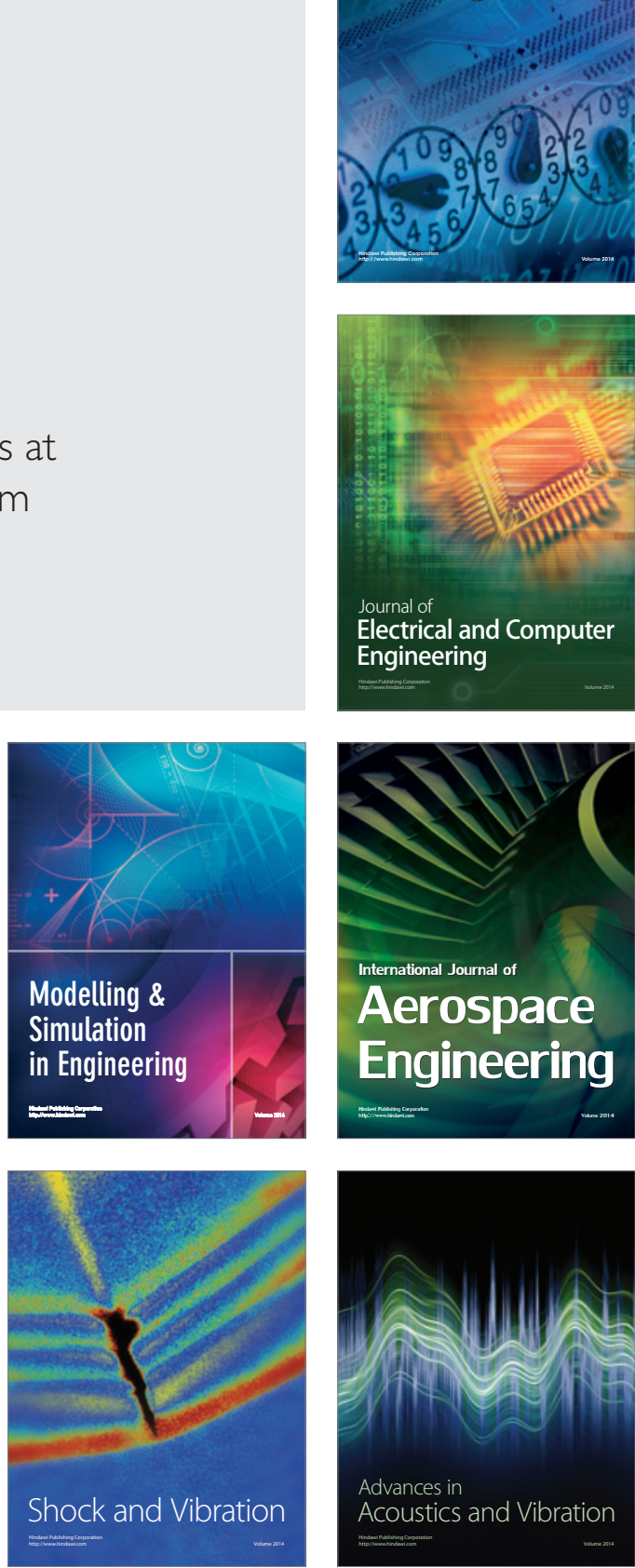